\title{
An attentional mechanism in the analysis of spatial frequency*
}

\author{
PATRICIA A. CARPENTER + and LEO GANZ \\ Stanford University, Stanford, California 94305
}

Sinusoidal gratings of various spatial frequencies were used as masking stimuli in a detection task and a vernier acuity task. The test stimuli were $1 \mathrm{cycle} / \mathrm{deg}$ square-wave gratings. The spatial frequency of the most effective mask was 1 cycle/deg for the detection task but 3 cycles/deg for the vernier acuity task. The different masking functions for the two tasks show that the visual system analyzes the square-wave stimulus into its various spatial-frequency components. Since the test stimulus was the same for both tasks, the different masking functions may be the result of an attentional mechanism that weighs the importance of the output from various spatial-frequency analyzers. Whether the information from a particular spatial-frequency analyzer is attended or not depends upon the task the visual system must perform.

The present experiment demonstrates that it is possible to interfere selectively with the mechanisms that detect the presence of a square-wave grating and the mechanisms that analyze the edges of the grating. The experimental strategy was to have Ss perform two tasks, a detection task and a vernier acuity task, described below. During each task, masking gratings were presented with the purpose of interfering with mechanisms that might contribute to performing either task. By measuring the interference caused by the masking gratings of various types, inferences could be made about the mechanisms that are utilized in the two tasks.

This experiment makes use of the fact that, mathematically, a square wave may be considered as the sum of components of different spatial frequencies. (Spatial frequency is the number of cycles of a wave per unit width.) By Fourier analysis, a square wave is the sum of sine waves whose frequencies are $1,3,5, \ldots$ times the frequency of the square wave, with amplitudes $4 / \pi, 4 / 3 \pi, 4 / 5 \pi, \ldots$ respectively; times the amplitude of the aquare wave. Thus, the first sinusoidal component, called the fundamental, has the greatest contrast (peak-to-trough difference) of all the components. The second and higher frequency components make up the sharp edges of the square wave.

Psychophysical evidence shows that the visual system codes spatial frequency (cf. Blakemore \& Sutton,

*This research was supported by a NSF predoctoral fellowship while the first author was at Stanford University and by NSF Was at Stantord University and by NSF 00364 awarded to the second author. We wish to thank Marcel Just and Greg Lockhead for their constructive comments. +Now at: Department of Psychology, Carnegie-Mellon University, Pittsburgh, Pennsylvania 15213.
1969; Blakemore \& Campbell, 1969; Campbell \& Robson, 1968). For instance, prolonged observation of a grating of a given spatial frequency will increase the threshold for perception of a subsequent test grating of that frequency (Pantle \& Sekuler, 1968). This effect diminishes with test gratings of decreasing similarity to the original grating. Presumably, during the prolonged observation period, mechanisms responsive to the spatial frequency of the grating are adapted out. Subsequent detection of a stimulus is more difficult if these adapted mechanisms are involved in the task. Thus, threshold elevation for a test grating indicates the same mechanisms stimulated by the adaptation grating are utilized in the perception of the test grating.

Not only is the visual system responsive to spatial frequency, but more importantly, the visual system seems to be sensitive to the various frequency components that make up the square wave. To distinguish square waves from sine waves, the visual system must have information about the abrupt edges of the square wave, i.e., information contained in the second and higher components. In fact, Campbell and Robion (1968) measured the contrast necessary to distinguish a square-wave grating from a sinusoidal grating which has the same frequency and amplitude as the fundamental component of the square wave. The contrast necessary to make this discrimination is equal to the contrast to just detect the second component of the square wave (the contrast to detect a sinusoidal grating at three times the frequency and $4 / 3 \pi$ the amplitude of the square wave). Thus, to distinguish a square-wave grating from a sinusoidal grating corresponding to the frequency and amplitude of the fundamental, the second component must be at threshold. This result implies that to obtain edge information, the visual system is responding to the second component of the square wave.

In the present experiment, there were two tasks, one of which depended on just detecting the square-wave grating, while the other depended on perceiving the edges. In the first task, called the detection task, an $S$ had to discriminate between the presence and absence of the square-wave grating. Here the $\mathbf{S}$ must be sensitive to the contrast between the light and dark stripes of the square-wave grating. Hence, detecting the fundamental should be more important for performing the task than detecting the higher components, because the fundamental accounts for most of the brightness contrast of the square wave. It follows that, in this task, interference should be maximal when the test stimulus is masked by a sinusoidal grating with the same frequency as the fundamental component. In the second task, a vernier acuity task, an $S$ had to discriminate the alignment from the misalignment of two vertical square-wave gratings, with one grating presented directly above the other. Here, presumably, the fundamental components of the top and bottom gratings are relatively useless in perceiving alignment or misalignment. The second and higher components should be more important than the fundamental because they carry the "edge information" of the square-wave grating - the information necessary to perceive the misalignment. Thus, in perceiving misalignment of two square-wave gratings, interference should be maximal when the test stimulus is masked by sinusoidal gratings of frequencies corresponding to the second and higher components.

\section{METHOD}

Subjects

The Ss were two paid Stanford students with 20-20 uncorrected vision. Both Ss were trained for $15-20 \mathrm{~h}$ on the two tasks before experimental data were collected.

\section{Apparatus and Procedure}

The masks were vertical sinusoidal gratings with spatial frequencies of $.75,1.00,1.94,3.06$, and 4.84 cycles/deg of visual angle. A sine-wave grating is a series of light and dark stripes, with gradual transition between light and dark that can be described by a sine wave. These masking gratings, $9.8 \mathrm{deg}$ wide $\mathrm{x}$ $9.8 \mathrm{deg}$ high, were generated on a Type 602 Tektronix oscilloscope with P11 (blue) phosphor and set at an average luminance of $2.2 \mathrm{~mL}$ when 


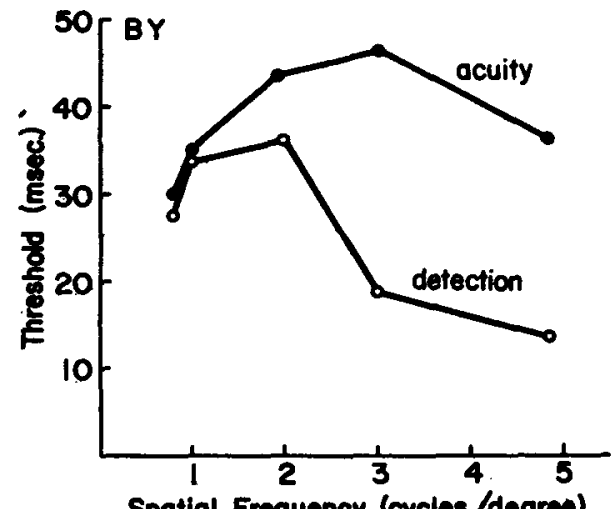

Spatial Frequency (cycles/degree)

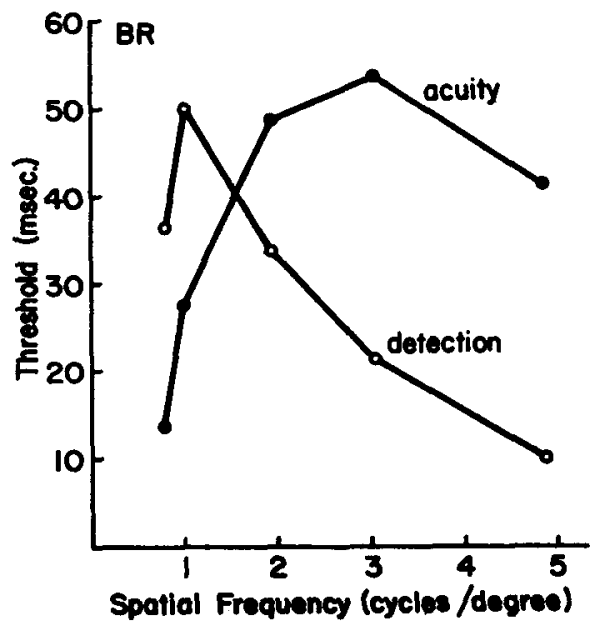

Fig. 1. Threshold (in milliseconds) for the detection and vernier acuity tasks as a function of the spatial frequency of the making grating.

z-axis modulation equalled $0 \mathrm{~V}$. The procedure used to generate the sinusoidal grids is described in detail by Campbell and Green (1965). A Heathkit audiogenerator controlled the spatial frequency of the grating and kept the contrast between the light and dark stripes at .81 for all masking gratings. That is, the peak-to-trough difference for the making gratines was kept constant, while frequency was varied. The test itimuli for both the detection and vernier acuity talks was 1 cycle/deg squnre-wave grating-vertical dark and light stripes. These square-wave grating were photorraphic negatives that were 7.7 deg wide x $7.2 \mathrm{deg}$ high, beck-illuminated and set at a distance of 22 in. from the $s$ in one arm of a two-channel Gerbrands tachistoccope with the oscilloscope display in the other arm. Colored filters matched the color of the test stimuli to that of the masking grating. For the vernier acuity task, a grating whose top and bottom halves were misaligned was presented on half the trials and a grating without misalignment on the other half. The misalignment was 0.05 deg of visual angle, and there was no separation between the top and bottom halves of the grating. The luminance of the bright bars in the grating was $2.9 \mathrm{~mL}$. The $\mathrm{S}$ simply reported whether the top and bottom halves were aligned or not. For the detection task, the aligned test grating was presented on half the trials and a homogeneous field of equivalent luminous flux on the other half. A gray filter was used on both kinds of detection trials in order to make the difficulty of the detection task more comparable to the difficulty of the vernier acuity task. The luminance of the bright bars of the grating was about $2.3 \mathrm{~mL}$. The S's task was to report whether he had seen the grating or the homogeneous field.

Both detection and vernier acuity thresholds were determined by the same method. The $\mathrm{S}$ initially adapted for $3 \mathrm{~min}$ by scanning across the sinusoidal grating between fixation points on the left and right sides located halfway up the grating. The test was presented with an exposure duration of $65 \mathrm{msec}$, and the $s$ responded whether it had been a positive or negative trial. After four such trials, the procedure was repeated at exposure durations successively decreasing by 5 msec, until the $S$ made two or three errors in the four trials at a single duration. Then he was presented with four more trials at that exposure time and, in ascending order, at all the previous exposure times. In all, there were eight randomly ordered positive and negative trials at each exposure duration. Threshold was considered to be the interval with $25 \%$ or more errors. In the few instances where the error rate fluctuated below 25\% and back again, threshold was the mean of the two time intervals with 25\% or more errors. Detection and vernier acuity terles were run on altemate days for 4 days each. For esch of the four terting seacions, the cerial order of the making grating was randomly determined and the same for both tenles.

Eneh trial was initiated when the $\mathbf{S}$ closed a switch, at which time the sinuscidal grating was changed to a uniform field of the same average luminance as the sinusoidal grating. Fifty millineconds later, the test timulus was flashed on the screen for a certain duration. The sinusoidal modulation returned 10 msec after stimulus offset and remained on until the next trial. The masking grating, although temporally separate, was kept spatially in phase with the test grating as much as possible. The intertrial interval was approximately $5 \mathrm{sec}$, with the entire session lasting about $10 \mathrm{~min}$. The $S$ rested $10 \mathrm{~min}$ between the different masking gratings.

\section{RESULTS AND DISCUSSION}

As the first hypothesis predicted, the masking grating of 1 cycle/deg interfered more with detection than did the higher frequency gratings. Figure 1 shows the results for the two Ss. For B.R., the most interference in the detection task occurred with a 1 cycle/deg mask. S B.Y. shows the most interference in the detection task for the 1.00 and 1.96 cycles/deg masks, which have quite comparable thresholds. In the vernier acuity task, both Ss show a maximum threshold with a masking grating of 3.06 cycles/deg. This is consistent with the hypothesis that the $S$ would utilize the "edge information" present in the second component to perform the vernier acuity task. As a baseline, detection and vernier acuity thresholds were measured after the Ss adapted to a homogeneous background equal in intensity to the peak of the sinusoidal masking gratings. The average of two threshold determinations for the vernier acuity task were $15 \mathrm{msec}$ for B.R. and 20 msec for B.Y. Both Ss performed better than criterion for threshold in the detection task with only a 5-msec exposure. Thus, the actual threshold is somewhat less than 5 msec, but the timing apparatus did not allow a finer determination of the detection threshold.

The threshold pattern for each $\mathbf{S}$ was extremely consistent across the 4 days. Four separate analyses for consigtency were done, for two Ss and two tasks. In each analysis, the thresholds for the five masking frequencies (excluding 0 cycles/deg) were ranked for each day, and a Friedman analysir of variance tested consintency acroes days. For S B.R., the ranking for the detection tak resulted in a $x^{2}=15.4$, df $=4$, $p<.01$, and for the vernier acuity tack, $x^{2}=14.6$ df $=4, p<.01$. For $s$ B.Y., the ranking for the detection task resulted in $x^{2}+11.7$, df $=4$, $p<.02$, and for the vernier scuity tank, $x^{2}=13.9$, df $=4, p<.02$. Therefore, the functions in Fig. 2 are significantly consiatent acrose days.

It is important to show that the making function is different for the detection and vernier scuity taaks when the test stimull are exactly the same. In the experiment described above, the stimuli were not identical. In the detection tak, there was an aligned grating, while in the vernier acuity task, there was an aligned grating on half the trials and an unaligned grating on the other half. To make the stimuli identical for both tasks, a control condition was run, consisting of the detection task with 
the unaligned grating as a test stimulus. The luminance of the bright bars was $2.3 \mathrm{~mL}$ for each grating. Since this control was run after the original experiment was completed, only one of the Ss (B.R.) was available for testing. After a small amount of practice, he was run in the detection task $-1 \mathrm{~h}$ a day for 2 days. Both the order of the masks and the order of the unaligned and aligned test gratings were counterbalanced. As Fig. 2 shows, the threshold patterns for the two kinds of test stimuli were virtually the same and duplicated the pattern in the original detection task. Thus, the spatial frequency of the most effective mask was different for the detection and vernier acuity tasks, even when the same gratings were used as test stimuli in the two tasks.

If a particular masking grating causes an increase in threshold, the mask may be stimulating the same mechanisms that are necessary to perform that task. Conversely, if a particular masking grating does not increase the threshold for a task, the mask may be stimulating mechanisms that are not necesary for performing that task. From these inferences, it follows that the fundamental component of the square wave contributes mainly to performance in the detection task and contributes very little to vernier acuity judgments. By contrast, the second component contributes mainly to vernier acuity judgments, and it contributes very little to detection of the square wave. Thu, not only can the visual system respond to the first and second component of a square wave, but also it can analyze these components somewhat independently.

The main experiment involved a 3-min adaptation period that terminated 50 msec before the test and reappeared 10 meec after the test. It is posible that the main effect was due to the prolonged adaptation period and that the backward and forward masking contributed little to the selective effects. To examine this hypothesis, part of the main experiment was repeated with the following modifications: The $S$ still scanned the sinusoidal grating for $3 \mathrm{~min}$ before the onset of the trials; however, the intervals of even illumination separating the test stimulus from the forward and backward masks were increased to eliminate any short-term masking effects of the sinusoidal gratings. When these interposed intervals before and after the test stimulus were increased to 100 msec, neither $\mathbf{S}$ showed much threshold elevation, while at 200 msec, all threshold elevation was eliminated. Thus, the results of the main experiment are primarily due to a

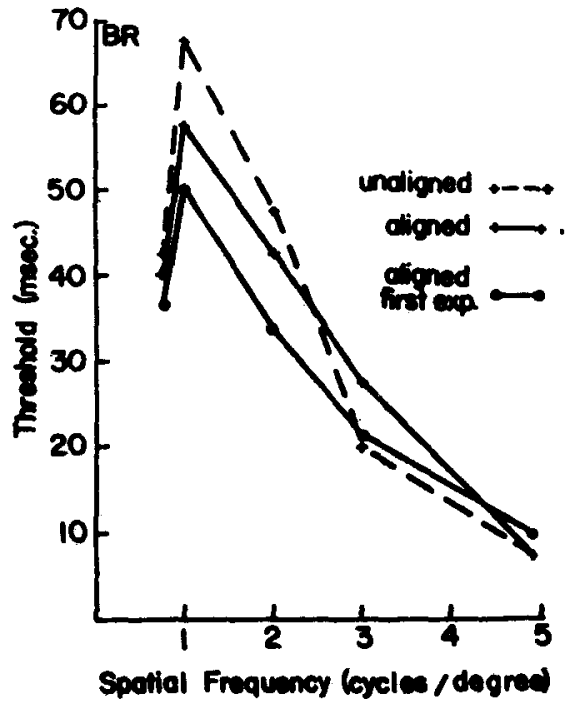

Fig. 2. Threshold (in milliseconds) for the detection task for S B.R., using, as test stimuli, gratings with and without the 0.05 -deg minalignment between the top and bottom halves of the prating. The regults for the aligned grating from the first experiment are the results from Fig. 1, shown here for comparieon with the other two detection tasks.

masking effect of the sinusoidal grating which doesn't extend much beyond 100 msec.

The effect of the masking grids has been discuseed in terms of how the masks interfere with the mechanisms that are responsive to different spatial frequencies. Physiological investigation suggest that these mechaniams have a neural besis. Reoearchen have located neurons at various levels of the cat's visual system that fire when grating of certain spatial frequencies are present on the retina (Campbell et al, 1969; Enroth-Cugell \& Robeon, 1966; Pollen et al, 1971). Similax neural mechanisms may exist in the human visual system and may be the source of spatial-frequency sensitivity (Campbell \& Maffei, 1970).

Some current theories of perception incorporate the notion of physiologically based feature analyzers as constructs to account for the encoding of external stimuli. Congruent with physiological data, there may be populations of analyzers that respond to a feature, where each member of such a population is optimally sensitive to a particular value of the feature. For example, among analyzers sensitive to spatial frequency, certain analyzers may respond optimally to one frequency and other analyzers respond optimally to other frequencies. The present experiment can be interpreted in terms of such a feature analysis model. Each spatial-frequency component in the square-wave may stimulate analyzers sensitive to that frequency. The amplitude of that component may determine some other characteristic of how the analyzers respond. For example, it is possible that the amplitude of the component may determine how many analyzers respond. A detection threshold for a particular component could be reached when a criterial number of analyzers tuned to that spatial frequency are stimulated.

In the detection task, the visual system utilizes information from all of the analyzers stimulated by the various components of the square wave. Previous research (Campbell \& Robson, 1964, 1968; Graham \& Nachmias, 1971) has shown that detection of a complex wave occurs when the component with the greatest amplitude reaches threshold. In the present experiment, the fundamental component of the square wave has the greatest amplitude. Interfering with analyzers responsive to the fundamental component forces the visual system to utilize information from analyzers responsive to higher frequency components-thus increasing the threshold. Conversely, interfering with analyzers responsive to the higher spatial frequencies doen't increase detection threahold because the threshold for these individual components is already higher than the threahold for the fundamental component.

The information from the various spatial-frequency analyzers is procesued differently in the vernier acuity task than in the detection task. In the vernier acuity tonk, the vimual yystem does not utilize information from the first component in the same way as it utilize information from the second component. Masking the first component does not increase the threshold as much as masking the second component. Thus, depending upon the task, some attentional mechanism may give more or less weight to information from the analyzers responsive to the various components of the square wave. In the acuity task, this attentional mechanism gives little weight to information about the fundamental, but relatively more weight to information about the edges-information from the analyzers responsive to the higher spatial frequencies. Interfering with the higher frequency analyzers that are important to vernier acuity performance increases the threshold. On the other hand, masking analyzers responding to the fundamental interferes with information that is 
fairly irrelevant, so the vernier acuity performance shows minimal interference. Thus, the visual system's response to various features of a stimulus depends not only on the system's sensitivity to those features, but also on the utility of perceiving them in a given task.

The data implicate some sort of attentional mechanism that alters the importance of information from various populations of spatial-frequency analyzers. This attentional mechanism can be conceived as either a passive or an active type of mechanism. It is possible that this attentional mechanism was established during training. For instance, perhaps the higher spatial-frequency analyzers became connected to the computing mechanism for the vernier acuity task but the low-frequency analyzers didn't. Thus, during the vernier acuity task, the attention process is automatic and passive. Another logical possibility is that the attentional process is activated on each vernier acuity trial. On each trial, this attentional mechanism may either allow information from the fundamental to enter into computing the response or disregard the information, depending upon the task. Thus, selective attention operates at the level of weighing information from spatial frequency analyzers differently for different tasks. No matter whether the attentional mechanism is active or passive, the different masking functions demonstrate that the visual system can separate out and disregard information from the fundamental component during the vernier acuity task, but can also utilize this information in the detection task.

\section{REFERENCES}

BLAKEMORE, C \& CAMPBELL, F. W. On the existence of neurones in the human visual system selectively sensitive to the orientation and size of retinal images. Journal of Physiology, 1969, 203, 237-260.

BLAKEMORE, C., \& SUTTON, P. Size adaptation: A new aftereffect. Science, $1969,66,245-247$.

CAMPBELL, F. W., COOPER, G. F., \& ENROTH-CUGELL, C. The spatial selectivity of the visual cells of the cat. Journal of Physiology, 1969, 203, 223-235.
CAMPBELL, F. W., \& GREEN, D. G. Optical and retinal factors affecting visual resolution. Journal of Physiology, 1965. 181, 576-593.

CAMPBELL, F. W., \& MAFFEI, L Electrophysiological evidence for the existence of orientation and size detectors in the human visual system. Journal of Physiology, 1970, 207 635-652.

CAMPBELL, F. W., \& ROBSON, J. G. Application of Fourier analysis to the modulation response of the eye. Journal of the Optical Society of America, 1964, $54,581 \mathrm{~A}$.

CAMPBELL, F, W, \& ROBSON, J. G. Application of Fourier analysis to the visibility of gratings. Journal of Physiology, 1968, 197, 551-566.

ENROTH-CUGELI, C. \& ROBSON, J. G. The contrast sensitivity of retinal ganglion cells of the cat. Journal of Physiology, 1966, 187, 517-552.

GRAHAM, N., \& NACHMIAS, J. Detection of grating patterns containing two spatial frequencies: A comparison of single-channel and multiple-channel models. Vision Research, 1971, 11, 251-259.

PANTLE, A., \& SEKULER, R Size-detecting mechanisms in human vision. Science, $1968,162,1146-1148$.

POLLEN, D. A., LEE, J. R., \& TAYLOR, J. H. How does the striate cortex begin the reconstruction of the visual world? Science, 1971, 173, 74-77.

(Accepted for publication November 24, 1971.) 